\title{
Estimation of Effective Permeability for Dumbbell-Shaped Magnetic Cores
}

\author{
Kunihisa Tashiro, Hiroyuki Wakiwaka, and Gen-ya Hattori
}

\begin{abstract}
The objective of this study is to provide suitable approximations of effective permeability for magnetic concentration cores. This core has dumbbell-shape, so that the magnetic flux is concentrated to the coil winding part of the core. It is well known that the demagnetizing factor strongly depends on the core shape which defines the effective permeability. Although several researchers have already proposed an approximation for a dumbbell-shaped core, it cannot take into account the flange part length. In this paper, a novel approximation for dumbbell-shaped core is proposed. It is based on the demagnetizing factor and correction terms related to the aspect ratio of both the flange and coil winding part. From experimental results, the validity of the approximation is confirmed.
\end{abstract}

Index Terms-Magnetic core, effective permeability, demagnetizing factor, magnetic concentration, dumbbell-shape

\section{INTRODUCTION}

$\mathrm{M}$ AGNETIC energy harvesting [1] has several applications related with both the energy harvesting and wireless power transmission [2]. Because the target is a weak magnetic field defined by ICNIRP2010 guideline, magnetic flux concentration methods are key techniques. In previous report, the importance of magnetic flux concentration core and coil was experimentally demonstrated [3]. The harvesting power is proportional to the square of the magnetic flux density, therefore it is very important to design the effective permeability of the core defined by the concentration ratio of magnetic flux density to the coil.

It is well known that the demagnetizing factor strongly depends on the core shape which defines the effective permeability. Because exact calculation of the demagnetization factor for ellipsoidal bodies exists [4], it is widely used for the estimation of the effective permeability for ellipsoidal core or relatively long rod core. Unfortunately, this estimation is not acceptable for the magnetic flux concentration core. The shape of the flange part, or magnetic flux concentration part, is a relatively long cylinder. And the cross section of the flange part is larger than that of the coil winding part. Several researchers

This work was supported in part by the JSPS KAKENHI, Scientific Research (C), Grant Number 25502001, 2013-2015.

Kunihisa Tashiro and Hiroyuki Wakiwaka are with the Spin Device Technology Center, Shinshu University, Nagano, Wakasato 3808553, Japan (e-mail: tashiro@shinshu-u.ac.jp, wakiwak@ shinshu-u.ac.jp).

Gen-ya Hattori, was graduated at the end of March 2014, from Shinshu University, Nagano, Wakasato 3808553, Japan. related to magnetic sensor have proposed an approximation for this core [5][6]. However, it cannot take into account the flange part length.

In this paper, a novel approximation for a dumbbell-shaped core is proposed. First of all, the estimation method of effective permeability for conventional rod-shaped cores are summarized. From a simplified model, an approximation is proposed. Compared with the previous approximation, a correction term is also introduced. It is based on the aspect ratio of both the flange and coil winding part. From experimental results, the validity of approximation is confirmed. This estimation method is also applicable for a magnetic sensor development.

\section{ESTIMATION OF EFFECTIVE PERMEABILITY}

Fig. 1 shows the magnetic flux concentration cores. The material of the cores is PC Permalloy. Compared with a conventional rod-shaped core, a magnetic concentration core can provide an amplification of an external magnetic field to the coil. Table 1 shows the specifications of the cores. In order to evaluate the effective permeability, coil were wound around the coil winding part. The shape of coil was Brooks coil [7] whose length, inner and outer diameter were $5 \mathrm{~mm}, 10 \mathrm{~mm}$ and $20 \mathrm{~mm}$, respectively. The number of coil turns was 500 with a wire of $0.2 \mathrm{~mm}$ in diameter.

The effective permeability, $\mu_{\text {eff }}$, is defined by the ratio of the mean magnetic field inside the core, $B_{\text {coil }}[\mathrm{T}]$, to the external magnetic field, $\mu_{0} H_{\mathrm{ext}}[\mathrm{T}]$ :

$$
\mu_{\text {eff }}=\frac{B_{\text {coil }}}{\mu_{\mathrm{o}} H_{\text {ext }}}=\frac{V_{\text {core_coil }}}{V_{\text {air_coil }}},
$$

where, $\mu_{0}[\mathrm{H} / \mathrm{m}]$ is the permeability in vacuum, $H_{\mathrm{ext}}[\mathrm{A} / \mathrm{m}]$ is the external magnetic field. $V_{\text {core_coil }}[\mathrm{V}]$ and $V_{\text {air_coil }}[\mathrm{V}]$ represent the induced voltage of the coil with or without the core, respectively.

\section{A. Conventional}

If the shape of the core is a conventional rod or plate, the value of $\mu_{\text {eff }}$ is defined by

$$
\mu_{\mathrm{eff}}=\frac{B_{\mathrm{coil}}}{\mu_{\mathrm{o}} H_{\mathrm{ext}}}=\frac{\mu_{r}}{1+\left(\mu_{r}-1\right) N},
$$


where, $\mu_{\mathrm{r}}$ is the relative permeability of the core, $N$ is the demagnetizing factor of the core. The value of $N$ can be expressed using the aspect ratio, $m$ :

$$
m=L / D
$$

where $L$ and $D$ are the outer length and diameter of the core, respectively. The value of $N$ can be roughly estimated by using the demagnetizing factor of the ellipsoidal bodies [8] as

$$
\left\{\begin{array}{lr}
N=\frac{1}{1-m^{2}}\left[1-\frac{m}{\sqrt{1-m^{2}}} \arccos m\right] & (m<1), \\
N=\frac{1}{3} & (m=1), \\
N=\frac{1}{m^{2}-1}\left[\frac{m}{\sqrt{m^{2}-1}} \operatorname{arccosh} m-1\right] & (m>1), \\
N=\frac{1}{m^{2}}(\ln 2 m-1) & (m>12) .
\end{array}\right.
$$

From (2), it is easy to understand that the value of $\mu_{\text {eff }}$ strongly depends on the value of $N$. In other words, the value of $\mu_{\text {eff }}$ is defined by the inverse value of $N$ when the value of $\mu_{\mathrm{r}}$ for the core is relatively large:

$$
\mu_{\mathrm{eff}}=\frac{1}{\frac{1}{\mu_{r}}+\left(1-\frac{1}{\mu_{r}}\right) N} \cong \frac{1}{N}
$$

Since demagnetizing fields are generally non-uniform, two demagnetizing factors should be defined: the fluxmetric and magnetometric demagnetizing factor. When a coil covering a core is concentrated in its central part, the fluxmetric demagnetizing factor should be used because the averaging area is the midplane of the core. If a winding covers a larger area remaining symmetrical relative to the core center, the magnetometric demagnetizing factor should be used because the averaging area is the entire volume. According to the reference in [9], M. A. Rozenblat proposed suitable approximations of effective permeability for both cases: $\mu_{\text {eff_center }}$ and $\mu_{\text {eff_volume: }}$

$$
\left\{\begin{array}{l}
\mu_{\text {eff_center }}=\frac{\pi L^{2}}{4 A\left(\ln \frac{k L}{a+b}-1\right)}, \\
\mu_{\text {eff_volume }}=\frac{\pi\left(L^{2}-0.25 l^{2}\right)}{4 A\left(\ln \frac{k L}{a+b}-1\right)},
\end{array}\right.
$$

where $A\left[\mathrm{~m}^{2}\right]$ is the cross-sectional area of the core, $l[\mathrm{~m}]$ is the coil length, $a[\mathrm{~m}]$ and $b[\mathrm{~m}]$ are orthogonal dimensions of the core, and $k$ is a coefficient as

$$
k=4-0.732 \times\left(1-e^{-5.5 a / b}\right) .
$$

To the best of the authors' knowledge, D-X Chen's group have reported several papers related the good approximation of demagnetizing factors for several ideal shapes: cylinder [8], square bars [10], and rectangular prism [11]. Aharoni's calculation is also one of famous approximation of demagnetizing factor for the rectangular prisms [12].

\section{B. Proposed}

Fig. 2 shows the schematic design of the concentration core. In order to improve the performance of induction magnetometer, several researchers also proposed similar core shape. Because of the differences in shape, the conventional approximation is not acceptable for a flux concentration core. From numerical computation results, C. Coillot's group [5] proposed an approximation of $\mu_{\text {eff }}$ for this kind of the core:

$$
\mu_{\mathrm{eff}}=\frac{\mu_{\mathrm{r}}}{1+\left(\mu_{\mathrm{r}}-1\right) N(L / D) \times(d / D)^{2}},
$$

where $N$ is the demagnetizing factor of the coil winding part. When the value of $\mu_{\mathrm{r}}$ is relative large, it could be expressed by

$$
\mu_{\mathrm{eff}}=\frac{1}{N} \times\left(\frac{D}{L}\right) \times\left(\frac{D}{d}\right)^{2} .
$$

This approximation consists of the demagnetizing factor of the coil winding part, $(1 / N)$, and two correction terms: the aspect ratio, $(D / L)$, and cross sectional ratio, $(D / d)^{2}$. The validity of the approximation was confirmed by experimental results for the flange shape of cone [5] and disk [6]. It was reported that the flange part ensured the good uniformity of magnetic flux density inside the coil winding part. In both cases, the length of the flange part is very short compared with that of the coil winding part. Therefore, this approximation was not taking into account the length of the flange part.

Although the exact calculation of the demagnetizing factor only exists for ellipsoidal bodies, this paper tries to provide an approximation and find the similarity. From (1), it could be expressed by following equations:

$$
\begin{aligned}
& \mu_{\mathrm{eff}}=\frac{B_{\text {flange }}}{\mu_{\mathrm{o}} H_{\mathrm{ext}}} \times \frac{B_{\text {coil }}}{B_{\text {flange }}}, \\
& B_{\text {core }}=\frac{\pi(D / 2)^{2}}{\pi(d / 2)^{2}} \times B_{\text {flange }}=\left(\frac{D}{d}\right)^{2} B_{\text {flange }} \quad[\mathrm{T}] \text {, } \\
& B_{\text {coil }}=B_{\text {core }}-\mu_{\mathrm{o}} H_{\mathrm{d}(\mathrm{core})}
\end{aligned}
$$

where $H_{\mathrm{d}}[\mathrm{A} / \mathrm{m}]$ is the demagnetizing field in the core, $B_{\text {flange }}$ [T], $B_{\text {coil }}[\mathrm{T}]$ and $B_{\text {core }}[\mathrm{T}]$ are the magnetic flux density of the flange, coil winding part, and at the connection surface between both parts, respectively. It is based on the conservation of the magnetic flux. In other words, all magnetic flux passes between the end and coil winding part. If the value of $\mu_{\mathrm{r}}$ is relatively large, the first term of right side in (10) can be expressed by 


$$
\frac{B_{\text {flange }}}{\mu_{\mathrm{o}} H_{\text {ext }}}=\frac{\mu_{\mathrm{r}}}{1+\left(\mu_{\mathrm{r}}-1\right) N_{\text {flange }}} \cong \frac{1}{N_{\text {flange }}} .
$$

Where $N_{\text {flange }}$ is the demagnetizing factor of the end part. Relationship between demagnetizing field and magnetic flux density of the core can be summarized by following equations:

$$
\begin{array}{ll}
H_{\mathrm{d}(\text { core })}=N_{\text {core }} M_{\text {core }} & {[\mathrm{A} / \mathrm{m}],} \\
B_{\text {core }}=\mu_{\mathrm{o}} H_{\text {core }}+\mu_{\mathrm{o}} M_{\text {core }} & {[\mathrm{T}],} \\
\mu_{\mathrm{r}}=B_{\text {core }} / \mu_{\mathrm{o}} H_{\text {core }} . &
\end{array}
$$

Where $N_{\text {core }}, M_{\text {core }}[\mathrm{A} / \mathrm{m}]$ are the demagnetizing factor and magnetization of the coil winding part, respectively. From (12), (14), (15) and (16), the magnetic flux density of the coil winding part is expressed by

$$
B_{\text {coil }}=\left[\left(1-N_{\text {core }}+\frac{N_{\text {core }}}{\mu_{\mathrm{r}}}\right)\right] B_{\text {core }} \quad[\mathrm{T}] .
$$

When the relative permeability is relatively high, the ratio of the $B_{\text {coil }} / B_{\text {core }}$ can be expressed by

$$
\frac{B_{\text {coil }}}{B_{\text {core }}}=\left[\left(1-N_{\text {core }}+\frac{N_{\text {core }}}{\mu_{\mathrm{r}}}\right)\right] \cong\left(1-N_{\text {core }}\right) .
$$

From (11), the ratio of the $B_{\text {coil }} / B_{\text {flange }}$ can be expressed by

$$
\frac{B_{\text {coil }}}{B_{\text {flange }}}=\left(1-N_{\text {core }}\right) \times\left(\frac{D}{d}\right)^{2} .
$$

From (10), (13), and (19), the value of $\mu_{\text {eff }}$ can be expressed by

$$
\mu_{\text {eff }}=\frac{1-N_{\text {core }}}{N_{\text {flange }}} \times\left(\frac{D}{d}\right)^{2} .
$$

It is apparent that the above described approximation is similar with the one described by (9). From several considerations, this paper adopts a correction term related to the aspect ratio:

$$
\mu_{\text {eff }}=\frac{1-N_{\text {core }}}{N_{\text {flange }}} \times\left(\frac{d}{l}\right) \times\left(\frac{D}{d}\right)^{2} .
$$

For calculation of both $N_{\text {core }}$ and $N_{\text {flange, }}$, (4) was used. The value of $m$ was $(l / d)$ for $N_{\text {core }}$, and $(\delta / D)$ for $N_{\text {flange, }}$, respectively.

\section{EXPERIMENT AND DISCUSSION}

Fig. 3 shows the schematic diagram of the experimental setup. An uniform magnetic field with magnetic flux density of $1 \mu \mathrm{T}$ at $60 \mathrm{~Hz}$ was generated by Simple-Cubic-3 coil system [13]. The measured value of $\mu_{\text {eff }}$ was calculated from the following equations:

$$
\begin{gathered}
\mu_{\mathrm{eff}}=\frac{V_{\text {core_coil }}}{V_{\text {air_coil }}}, \\
V_{\text {air_coil }}=2 \pi f n A \times \mu_{0} H_{\text {ext }},
\end{gathered}
$$

where $V_{\text {core_coil }}[\mathrm{V}]$ is the induced voltage of the coil measured with a lock-in amplifier (5610B, NF corp.), $V_{\text {air_coil }}[\mathrm{V}]$ is calculated from Faraday's low of induction, $f[\mathrm{~Hz}]$ is the frequency $(=60 \mathrm{~Hz}), n$ is the number of coil turns, $A\left[\mathrm{~m}^{2}\right]$ is mean cross section of the coil and $\mu_{0} H_{\text {ext }}[\mathrm{T}]$ is the external magnetic flux density $(=1 \mu \mathrm{T})$.

Fig. 4 shows the value of $\mu_{\text {eff }}$ as a function of the value of $\delta$. The plots represent the measurement results, line and dotted line represent the calculation results from (21) and (9), respectively. From experimental results, the validity of the proposed approximation was successfully confirmed. The relative values of estimation error were less than $10 \%$.

It was found that the calculation results from (9) were not agreed with the experimental results. One of the possible reason is acceptable aspect ratio of $(L / D)$ because both approximation contain the correction terms. In order to check the acceptable aspect ratio for the proposed approximation, FEM analysis was conducted.

Fig. 5 shows the value of $\mu_{\text {eff }}$ as a function of the value of $L / D$. Filled plots represent the measured results, and line represents the calculation results from (21). Open plots represent the FEM analysis results with JMAG-Designer Ver. 12.0.2. Although the ratio of a coil part length, coil part diameter and flange diameter was fixed to 1:2:4, several similar models were tested for the FEM analysis. The FEM analysis indicated that proposed approximation could be acceptable when the $L / D$ was less than 2.25 . It could be also useful to design an magnetometer having short length of the core.

\section{CONCLUSION}

This paper presented an approximation for the effective permeability of a magnetic flux concentration core which has dumbbell-shape. This approximation contained two demagnetizing factors: the flange and coil winding part of the core; two correction term: the aspect ratio and cross sectional ratio. From both the experimental and FEM analysis results, it was confirmed that the proposed approximation could be acceptable when the $L / D$ was less than 2.25 .

\section{ACKNOWLEDGMENT}

The authors thank to Mr. Syoichiro Shimada for helping the confirmation by FEM analysis. This work was supported in part by the JSPS KAKENHI, Scientific Research (C), Grant Number 25502001, 2013-2015.

\section{REFERENCES}

[1] K. Tashiro, H. Wakiwaka, S. Inoue, and Y. Uchiyama, "Energy harvesting of magnetic power-line noise", IEEE Trans. Magn., vol. 47, pp. 4441-4444, 2011.

[2] K. Tashiro, H. Wakiwaka, and Y. Uchiyama, "Theoretical design of energy harvesting module or wireless power transmission receiver using magnetic field of $0.2 \mathrm{mT}$ at $60 \mathrm{~Hz}$, Journal of Energy and Power Engineering, vol. 7, pp. 740-745, 2013. 
[3] K. Tashiro, G. Hattori, and H. Wakiwaka, "Magnetic flux concentration method for magnetic energy harvesting module", EPJ Web of Conferences, 40, 06011, 2013.

[4] J. A. Osborn, "Demagnetizing factors of the general ellipsoid.", Phys. Rev., vol. 67, pp. 351-357, 1945.

[5] C. Coillot, J. Moutoussamy, P. Leroy, G. Chanteur, and A. Roux, "Improvements on the design of search coil magnetometer for space experiments", Sensor Letters, vol. 5, pp. 167-170, 2007.

[6] E. Paperno, and A. Grosz, "A miniature and ultralow power search coil optimized for a $20 \mathrm{mHz}$ to $2 \mathrm{kHz}$ frequency range", Journal of Applied Physics, vol. 105, 07E708, 2009.

[7] F. W. Grover, "Inductance Calculations", Dover Phenix Editions, pp. 94-113, 2004

[8] D.-X. Chen, E. Pardo, and A. Sanchez, "Fluxmetric and magnetometric demagnetizing factors for cylinders", Journal of Magnetism and Magnetic Materials, vol. 306, pp. 135-146, 2006.

[9] G. Musmann and Y. Afanassiev, "Fluxgate magnetometers for space research", BoD, pp. 21-31, 2010.

[10] E. Pardo, D.-X. Chen, and A. Sanchez, "Demagnetizing factors for square bars", IEEE Trans Magn., vol. 40, pp. 1491-1498, 2004.

[11] D.-X. Chen, E. Pardo, and A. Sanchez, "Demagnetizing factors for rectangular prisms ", IEEE Trans Magn., vol. 41, pp. 2077-2088, 2005.

[12] A. Aharoni, "Demagnetizing factors for rectangular ferromagnetic prisms", J. Appl. Phys, vol. 83, pp. 3432-3434, 1998.

[13] K. Tashiro, A. Matsuoka, and H. Wakiwaka, "Simple-Box-9 coil system: A novel approach to design of a square coil system for producing uniform magnetic fields," Materials Science Forum, vol. 670, pp. 275-283, 2011.

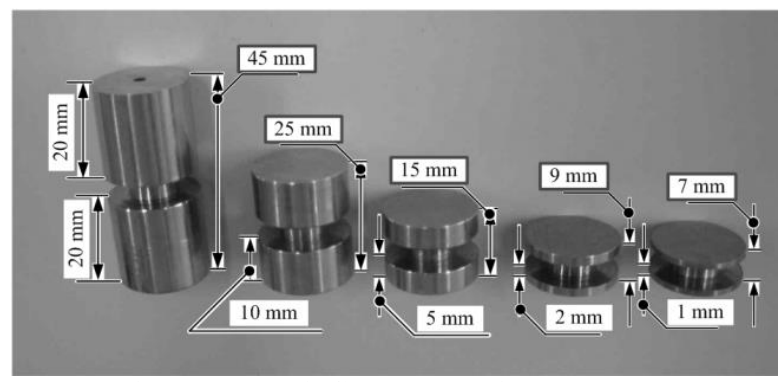

Fig. 1. Magnetic flux concentration cores.

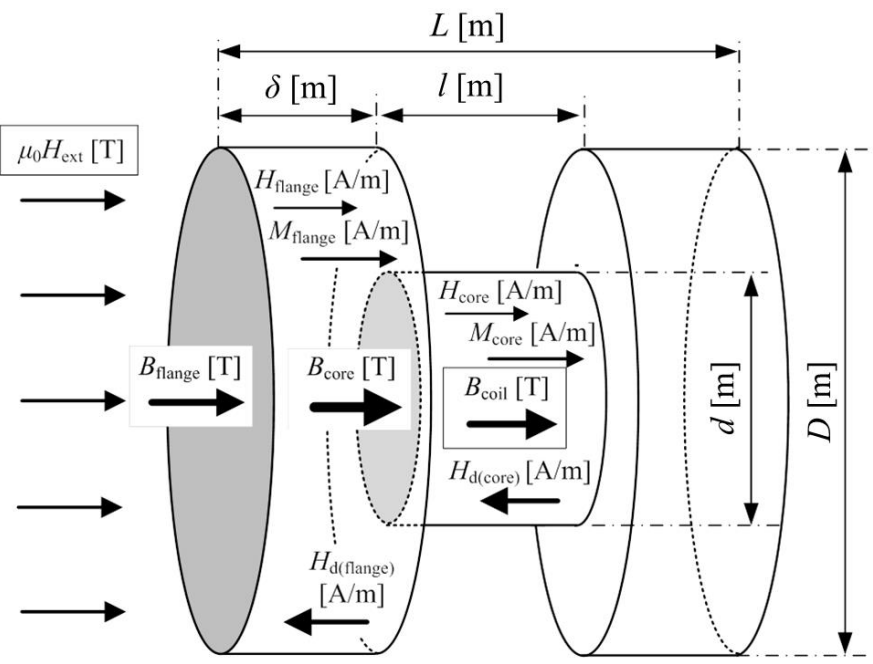

Fig. 2. Schematic design of the magnetic concentration core.

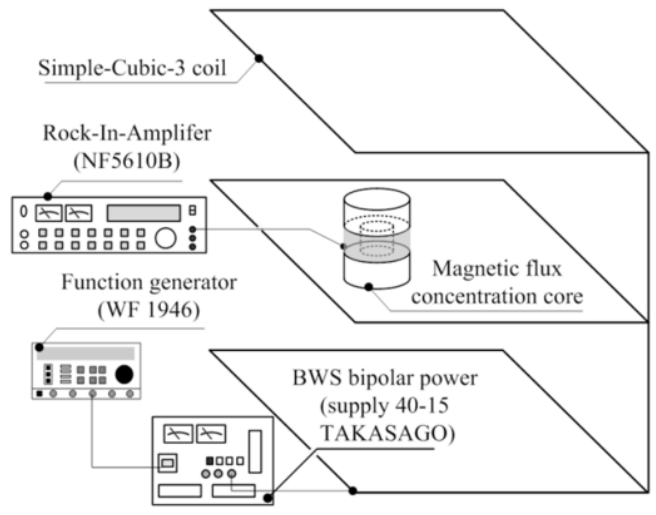

Fig. 3. Schematic design of the experimental setup.

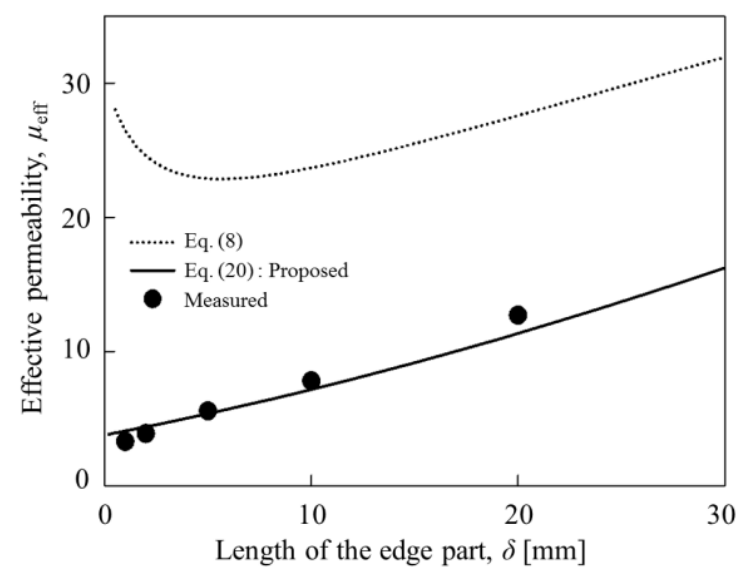

Fig. 4. Effective permeability as a function of the flange part length. The plot represents the measurement results, line and dotted line represent the calculation results from (21) and (9), respectively.

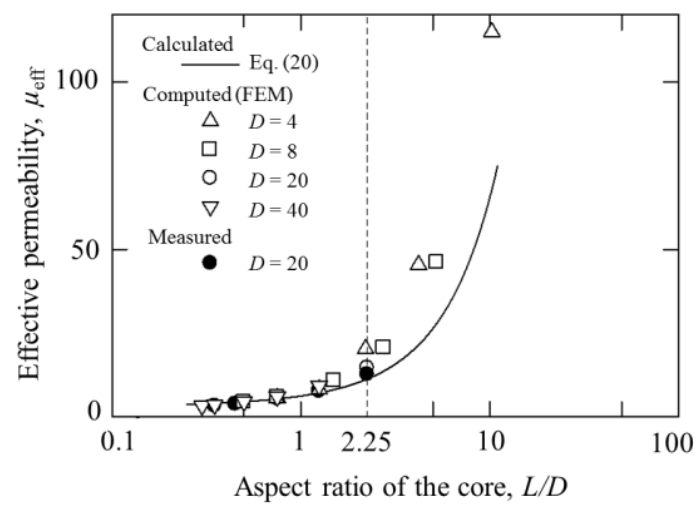

Fig. 5. Effective permeability as a function of the aspect ratio. Line represents the calculation results from (21), filled and open plots represent the measurement and FEM analysis results, respectively.

TABLE I

SPECIFICATIONS OF THE MAGNETIC FLUX CONCENTRATION CORES

\begin{tabular}{lc}
\hline \hline Parameter & Value \\
\hline coil length, $l[\mathrm{~mm}]$ & 5 \\
inner diameter, $d[\mathrm{~mm}]$ & 10 \\
outer diameter, $D[\mathrm{~mm}]$ & 20 \\
flange length, $\delta[\mathrm{mm}]$ & $1,2,5,10,20$ \\
material & PC Permalloy (Nilaco Inc.) \\
\hline \hline
\end{tabular}

